\title{
Management of Alveolar Cleft
}

\author{
Hyunwoo Kyung, \\ Nakheon Kang \\ Department of Plastic and Reconstructive \\ Surgery, Chungnam National University \\ School of Medicine, Daejeon, Korea
}

No potential conflict of interest relevant to this article was reported.
The alveolar cleft has not received as much attention as labial or palatal clefts, and the management of this cleft remains controversial. The management of alveolar cleft is varied, according to the timing of operation, surgical approach, and the choice of graft material. Gingivoperiosteoplasty does not yet have a clear concensus among surgeons. Primary bone graft is associated with maxillary retrusion, and because of this, secondary bone graft is the most widely adopted. However, a number of surgeons employ presurgical palatal appliance prior to primary alveolar bone graft and have found ways to minimize flap dissection, which is reported to decrease the rate of facial growth attenuation and crossbite. In this article, the authors wish to review the literature regarding various advantages and disadvantages of these approaches.

Keywords: Cleft lip / Cleft palate/ Alveolar bone grafting / Periosteum

\section{INTRODUCTION}

Alveolar cleft reconstruction using bone graft was first reported by von Eiselsberg [1] in 1901, and Drachter [2] reported such method using tibial bone graft in 1914. Despite these early reports, alveolar cleft did not receive as much attention as did labial and palatal clefts for a time. It was only after World War II that alveolar repair began to appear again in the literature. In 1958, Schrudde and Stellmach [3] reported primary repair of alveolar graft using bone graft in infants. In the subsequent decade, Skoog [4] introduced a method by which he reported successful repair of alveolar defect via primary gingivoperiosteoplasty (GPP), albeit without bone graft.

Since the Post-WWII Era, the most commonly used method of alveolar reconstruction has been early primary closure with bone graft. Several decades of primary bone grafts have demonstrated significant disadvantages, and secondary bone graft reconstruction has become a legitimate competing standard. In this context,

Correspondence: Nakheon Kang

Department of Plastic and Reconstructive Surgery, Chungnam National University Hospital, Chungnam National University School of Medicine, 282 Munhwa-ro, Jung-gu, Daejeon 35015, Korea

E-mail:nhk488@cnu.ac.kr

Received August 5, 2015 / Revised August 6, 2015 / Accepted August 6, 2015 a number of craniofacial centers continue to repair alveolar clefts primarily (bone graft or GPP) with certain modifications to reduce the incidence of midface retrusion and anterior corssbite.

\section{EMBRYOLOGY}

The palate develops from the frontonasal and maxillary prominences between 4 and 12 weeks of gestation. During gestational weeks 4 to 7 , the primary palate originates from the median palatine process, which itself originated from frontonasal prominence. The lip, alveolus, and hard palate anterior to the incisive foramen all share this origin in development. During this period, disruptions of palatal development process can result in primary palate clefting.

The secondary palate develops from the lateral palatine processes originating from the maxillary prominences between 7 and 12 weeks, and the hard palate posterior to incisive foramen and the soft palate are developmental products of this process. As such, disruptions during this time will result in secondary palate clefts.

The alveolus is a component of the primary palate, and alveolar cleft results from mal-development of the frontonasal prominence. As such, it is most closely related to cleft lip and cleft palate. 
Generally, alveolar clefts are located between the lateral incisor and canine [5].

\section{TREATMENT OBJECTIVES}

Alveolar cleft repair has both functional and aesthetic restoration as its goals. The functional goal includes closure of nasolabial fistula, creation of a stable and continuous maxillary dental arch, improved support of teeth adjacent of the cleft site, allowance for eruption of teeth into the cleft site, provision of unrestricted orthodontic movement, facilitation of oral hygiene, and speech improvement. Persistent nasolabial fistula will allow chronic nasal regurgitation, which leads to chronic inflammation and nasal discharge.

The aesthetic goal of alveolar cleft repair consists of augmentation of the pyriform region and the creation of a cosmetically pleasing dental arch and dentition. Augmentation of hypoplastic pyriform region can improve alar base support and asymmetry [6]

\section{TIMING OF REPAIR}

The timing of operative intervention for alveolar cleft is controversial. GPP is usually performed between 3 and 6 months of age. Primary bone grafting is performed between birth and 2 years of age, and the alveolar cleft can be repaired simultaneously with cleft lip or cleft palate repair. Secondary bone grafting can be divided into early, conventional, and late variants. Early secondary repair is commenced after complete eruption of primary dentition and before the eruption of permanent dentition-between 2 to 6 years. Conventional (transitional) secondary repair is performed during the mixed dentition stage between 6 and 12 years. Late secondary (tertiary) repair is performed after eruption of all permanent dentition [6].

\section{GINGIVOPERIOSTEOPLASTY}

GPP removed soft tissue barriers against alveolar fusion and uses the gingiovoperiosteal flaps to create a soft tissue tunnel, which is designed to allow bone conduction from both ends of the ridge. While primary GPP was introduced in 1969 by Skoog [7], it was not widely welcomed because of stunted facial bone growth. In 1980, Millard [8] reported the use of Latham appliance in conjunction with GPP. His solution to the problem of stunted facial growth was presurgical orthopedics, periosteoplasty, and lip adhesion [8]. The active mechanical presurgical approximation of the alveoli did reduce the need for subperiosteal dissection but was associated with maxillary vertical growth disturbance (40\%$42 \%)$ and cross bite (100\%).

Subsequent to this, Grayson et al. [9] reported a more passive molding process using nasoalveolar molding, and this method has been widely adopted as a presurgical orthodontic alignment method prior to GPP repair of alveolar clefts.

In GPP, the roof is created using the nasal flaps with the floor creating using the oral flaps. The anterior lower wall is created with labial flaps and the anterior superior wall is created with the lip mucosa. Thus, the chamber is sealed nasally, orally, and labially by mucoperiosteal flaps.

GPP for alveolar cleft has the advantage of not requiring a secondary bone graft, but can induce iatrogenic facial growth restriction and malocclusion. According to the 1999 study from the New York University (NYU) group, GPP was able to induce bone growth in $80 \%$ of cases but still required secondary bone graft in $40 \%$ of patients [10]. This need for secondary bone graft was replicated in a study out of Chang Gung Memorial Hospital, in which $28 \%$ of patients required secondary bone graft [11]. In 2008, Matic and Power [12] had reviewed various methods of alveolar repair patients using objective scales. The authors reported that the secondary bone graft-only management was superior to GPP with or without secondary bone graft, and observed that bone formation was lesser quality and in poorer location in patients who received GPP-only management. However, Sato et al. [13] compared the same methods of alveolar graft repair using Long's rating scale and found that their experience did not agree with that of Matic et al. These authors reported that GPP-alone was associated with highest rate of success and that secondary bone graft alone was associated with the worst outcomes [13]. Thus, outcomes following GPP remains controversial.

The NYU group reported that GPP was not associated with adverse effect on facial growth [14]. However, Hsieh et al. [15] was 
able to detect 2.1 and $2.9 \mathrm{~mm}$ of deficiencies in sagittal maxillary and maxillary alveolar lengths, respectively. Matic and Power [12] reported that maxillary vertical height was best in patients who had received salvage bone grafting after failed GPP. To evaluate such discrepancy in GPP outcomes, rodent model experiements are underway, using recombinant human bone morphogenetic protein (rhBMP) and other novel approaches to inducing bone conduction [16].

\section{PRIMARY ALVEOLAR BONE GRAFTING}

The purpose of primary bone graft is to close the nasobuccal or perialveolar fistula at an earlier age, to promote closure of primary palatal cleft, and to elevate alar floor to assist in lateral soft tissue growth. The contemporary approach of primary bone graft repair uses Rosenstein and Dado protocol, in which presurgical orthognathics prevents medial migration of the buccal segment. The lip cleft is repaired 6 to 8 weeks of age, and appropriate tension in muscle repair helps with alveolar molding. After the labial repair, the passive palatal appliance is used to prevent alveolar collapse. The alveolar segments are either allowed to come into contact with each other or are separated by 1 to $2 \mathrm{~mm}$. Primary bone graft is performed around 4 to 5 months of age.

\section{Source of bone graft material}

Rib bone is used as a donor graft in Rosenstein's primary bone grafting protocol. Possible complications include bone resorption, atelectasis, pneumothorax, scarring, and persistent pain. Other investigators have reported difficulties with dental movement and eruption during orthodontic treatment.

\section{Surgical procedure}

The sixth rib is exposed via a 3-cm skin incision. The periosteum is incised in an H-shaped fashion, and the bone is harvested through this window. The bony gap at the donor site spontaneously regenerates in few weeks. The harvested rib is split into anterior and posterior halves, of which the posterior half is pulverized.
The posterior lining is repaired using mucoperiosteal flaps and the rib strut is fitted into the cleft space. The bone fragments are then filled into the empty space. The buccal mucosa is mobilized inferiorly in a $\mathrm{V}$ shape, and the posterior lining and inferior edges are sutured together. The remaining mucosa is sutured to the anterior edge.

Rosenstein et al. [17] has reported good arch form and minimal cross bite in a long-term follow up of patients who have undergone primary bone repair. These patients demonstrated neither inhibition of facial growth nor evidence of maxillary segment collapse. Approximately $22 \%$ of patients from that series required orthognathic operation, which is similar to the $25 \%$ figure reported by Ross [18].

\section{CONCLUSION}

Depending on the timing of surgical intervention, alveolar cleft has been managed with various methods. GPP continues to require further evaluation of the outcomes. Primary bone graft stabilizes the dental arch at an earlier age and induces tooth migration and eruption. Facial growth attenuation had prevented widespread adoption. Certain craniofacial centers have modified primary bone graft repair. The studies coming out of these centers found that no significant difference exists in facial growth attenuation between their modified method and secondary bone grafting, and also reported that primary bone grafting can promote solid foundation for further soft tissue growth. Primary bone graft is best performed after labial but prior to palatal repair, around 4 to 5 months, and presurgical palatal appliance can increase the rate of successful alveolar alignment. A review of the alveolar cleft literature is ambivalent on whether GPP or primary bone graft provides advantages over secondary bone graft. Further research is needed to further develop a more appropriate method of alveolar cleft treatment.

\section{REFERENCES}

1. von Eiselsberg F. Zur technik der uranoplastik. Arch Klin Chir 1901;64:509-29.

2. Drachter R. Die gaumenspalte und deren operative behandlung. Dtsch Zschr Chir 1914;131:1-89. 
3. Schrudde J, Stellmach R. Primary osteoplasty of defects of the inferior maxillary arch in cleft palate and harelip in infants; preliminary report. Zentralbl Chir 1958;83:849-59.

4. Skoog T. The use of periosteum and Surgicel for bone restoration in congenital clefts of the maxilla: a clinical report and experimental investigation. Scand J Plast Reconstr Surg 1967;1:113-30.

5. Bajaj AK, Wongworawat AA, Punjabi A. Management of alveolar clefts. JCraniofac Surg 2003;14:840-6.

6. Daw JL Jr, Patel PK. Management of alveolar clefts. Clin Plast Surg 2004;31:303-13.

7. Skoog T. Repair of unilateral cleft lip deformity: maxilla, nose and lip. Scand J Plast Reconstr Surg 1969;3:109-33

8. Millard D. Presurgicla maxillary orthopedics. In: Millard DR, editor. Cleft craft: the evolution of its surgery. Boston: Little, Brown; 1980. p. 263-98.

9. Grayson BH, Santiago PE, Brecht LE, Cutting CB. Presurgical nasoalveolar molding in infants with cleft lip and palate. Cleft Palate Craniofac J 1999;36:486-98.

10. Santiago PE, Grayson BH, Cutting CB, Gianoutsos MP, Brecht LE, Kwon SM. Reduced need for alveolar bone grafting by presurgical orthopedics and primary gingivoperiosteoplasty. Cleft Palate Craniofac J 1998;35:77-80.

11. Wang YC, Liao YF, Chen PK. Outcome of gingivoperiosteoplasty for the treatment of alveolar clefts in patients with unilateral cleft lip and palate. Br J Oral Maxillofac Surg 2013;51:650-5.

12. Matic DB, Power SM. Evaluating the success of gingivoperiosteoplasty versus secondary bone grafting in patients with unilateral clefts. Plast Reconstr Surg 2008;121:1343-53.

13. Sato Y, Grayson BH, Garfinkle JS, Barillas I, Maki K, Cutting CB. Success rate of gingivoperiosteoplasty with and without secondary bone grafts compared with secondary alveolar bone grafts alone. Plast Reconstr Surg 2008;121:1356-67.

14. Wood RJ, Grayson BH, Cutting CB. Gingivoperiosteoplasty and midfacial growth. Cleft Palate Craniofac J 1997;34:17-20.

15. Hsieh CH, Ko EW, Chen PK, Huang CS. The effect of gingivoperiosteoplasty on facial growth in patients with complete unilateral cleft lip and palate. Cleft Palate Craniofac J 2010;47:439-46.

16. Nguyen PD, Lin CD, Allori AC, Schachar JS, Ricci JL, Saadeh PB, et al. Scaffold-based rhBMP-2 therapy in a rat alveolar defect model: implications for human gingivoperiosteoplasty. Plast Reconstr Surg 2009;124:1829-39.

17. Rosenstein S, Kernahan D, Dado D, Grasseschi M, Griffith BH. Orthognathic surgery in cleft patients treated by early bone grafting. Plast Reconstr Surg 1991;87:835-92.

18. Ross RB. Treatment variables affecting facial growth in complete unilateral cleft lip and palate. Cleft Palate J 1987;24:5-77. 